\title{
Nanostructured polyurethane-poly-lactic- co-glycolic acid scaffolds increase bladder tissue regeneration: an in vivo study
}

This article was published in the following Dove Press journal:

International Journal of Nanomedicine

27 August 2013

Number of times this article has been viewed

\author{
Chang Yao' \\ Matt Hedrick' \\ Gyan Pareek ${ }^{2}$ \\ Joseph Renzulli² \\ George Haleblian ${ }^{2}$ \\ Thomas JWebster ${ }^{3}$ \\ 'Nanovis LLC, West Lafayette, \\ IN, ${ }^{2}$ Section of Minimally Invasive \\ Urology, Alpert Medical School, \\ Brown University, Providence, RI, \\ ${ }^{3}$ Department of Chemical Engineering \\ and Program in Bioengineering, \\ Northeastern University, Boston, \\ MA, USA
}

\begin{abstract}
Although showing much promise for numerous tissue engineering applications, polyurethane and poly-lactic-co-glycolic acid (PLGA) have suffered from a lack of cytocompatibility, sometimes leading to poor tissue integration. Nanotechnology (or the use of materials with surface features or constituent dimensions less than $100 \mathrm{~nm}$ in at least one direction) has started to transform currently implanted materials (such as polyurethane and PLGA) to promote tissue regeneration. This is because nanostructured surface features can be used to change medical device surface energy to alter initial protein adsorption events important for promoting tissue-forming cell functions. Thus, due to their altered surface energetics, the objective of the present in vivo study was to create nanoscale surface features on a new polyurethane and PLGA composite scaffold (by soaking the polyurethane side and PLGA side in $\mathrm{HNO}_{3}$ and $\mathrm{NaOH}$, respectively) and determine bladder tissue regeneration using a minipig model. The novel nanostructured scaffolds were further functionalized with IKVAV and YIGSR peptides to improve cellular responses. Results provided the first evidence of increased in vivo bladder tissue regeneration when using a composite of nanostructured polyurethane and PLGA compared with control ileal segments. Due to additional surgery, extended potentially problematic healing times, metabolic complications, donor site morbidity, and sometimes limited availability, ileal segment repair of a bladder defect is not optimal and, thus, a synthetic analog is highly desirable. In summary, this study indicates significant promise for the use of nanostructured polyurethane and PLGA composites to increase bladder tissue repair for a wide range of regenerative medicine applications, such as regenerating bladder tissue after removal of cancerous tissue, disease, or other trauma.
\end{abstract}

Keywords: polyurethane, poly-lactic-co-glycolic acid, nanotechnology, nanostructured features, in vivo

\section{Introduction}

Due to optimal mechanical properties but a frequent lack of suitable cytocompatibility, researchers have suggested several techniques to improve polyurethane (PU) for numerous tissue engineering applications..$^{1-5}$ Similarly, due to its optimal degradation properties but lack of suitable cytocompatibility, various techniques have been proposed to improve poly-lactic-co-glycolic acid (PLGA) for tissue engineering applications..$^{1-5}$ Whereas PU is hydrolytically stable, PLGA degrades in aqueous environments due to ester bond breakage. Importantly though, $\mathrm{PU}$ has been shown to degrade in vivo due to the presence of ether linkages in PU soft segments susceptible to chemical degradation. Ether and ester bond breakage allows both PU and PLGA, respectively, to be easily chemically and structurally modified to improve cellular responses. ${ }^{3-5}$
Correspondence: Thomas J Webster Department of Chemical Engineering, Northeastern University, Boston, MA 02115, USA

$\mathrm{Tel}+\mathrm{I} 6173736585$

Fax + I 6173736588

Email th.webster@neu.edu 
Thapa et al were the first to treat PU and PLGA separately with various concentrations of $\mathrm{HNO}_{3}$ and $\mathrm{NaOH}$, respectively, for selected periods of time to promote nanoscale surface features to enhance in vitro bladder cell responses. ${ }^{1,2}$ Miller et al followed and demonstrated greater in vitro vascular cell responses while Vance et al provided evidence of decreased in vitro fibroblast responses leading to decreased prolonged fibrous tissue growth on such nanostructured films compared with conventional PU and PLGA films ${ }^{6,7}$ Kay et al also provided evidence of greater function of chondrocytes (cartilageforming cells) on nanostructured polymers compared with conventional polymers and polymer composites. ${ }^{8}$ All of the aforementioned studies used cell adhesion and proliferation as indicators of enhanced cell function.

In an attempt to understand the properties of $\mathrm{HNO}_{3}$ treated PU and $\mathrm{NaOH}$-treated PLGA that enhanced cellular responses, Thapa et al and Miller et al created separate PU and PLGA molds of conventional and $\mathrm{HNO}_{3}$-etched $\mathrm{PU}$ and $\mathrm{NaOH}$-etched PLGA, respectively (thereby eliminating chemical changes and studying only nanometer roughness changes), and found similar increases in in vitro bladder and vascular cell functions, respectively, on the nanostructured molds compared with conventional molds. ${ }^{1,2,6,7}$

Thus, to date, the most promising consequence of $\mathrm{HNO}_{3}$ treatment of PU and $\mathrm{NaOH}$ treatment of PLGA has been the creation of nanometer surface roughness promoting selected cell responses important for the regeneration of numerous tissues, but no data have been provided to date concerning the ability of these nanostructured polymers to regenerate bladder tissue in vivo. Moreover, several studies have indicated $^{9-15}$ that $\mathrm{PU}$ does not possess desirable biodegradability or mechanical properties compared with natural bladder tissues. ${ }^{1-5}$ For this and all of the above reasons, the objective of the present study was to create novel graded PLGA-PU scaffolds with nanoscale surface roughness and characterize their ability to regenerate bladder tissue using a well established minipig model.

\section{Materials and methods Materials}

Grafts were produced using a salt leaching method to achieve $80 \%-90 \%$ target porosity and the continuously graded composition as shown in Figure 1. Specifically, the scaffolds were created through a salt leaching method to introduce pores into the polymers using $150-250 \mu \mathrm{m}$ diameter salt $(\mathrm{NaCl})$ particles (Sigma, St Louis, MO, USA) at a 10:90 polymer to salt ratio by weight. To create layers of increasing amounts of PLGA on PU-PLGA composites, layers of the next increasing weight percentage of PLGA to PU were placed by pipette on top of the lesser percentage of PLGA to PU ratio. This was done for every $20 \mathrm{wt} \%$ of PLGA to PU until the composite formed was complete PU ( $0 \%$ PLGA) to complete PLGA ( $0 \%$ PU). The PLGA used was $75: 25 \mathrm{wt} \%$ (PLA to PGA, $12-16.5 \times 10^{3}$ molecular weight; Polysciences Inc, Warrington, PA, USA) and the PU used was Tecoflex ${ }^{\circledR}$ (medical grade SG80A, 90,000-140,000 kDa Noveon Inc, Minneapolis, MN, USA). Each polymer was dissolved in 1,1,1,3,3,3-hexafluoro-2-propanol at a concentration of $100 \mathrm{mg} / \mathrm{mL}$. After forming each layer of the scaffold, vacuum drying was used followed by rinsing with water to remove the salt (and thereby create pores). To induce nanoscale topologies on both the PLGA and PU layers, the scaffolds were exposed to $1.0 \mathrm{~N} \mathrm{NaOH}$ on the PLGA side and then flipped and exposed to $10 \mathrm{~N} \mathrm{HNO}_{3}$ on the PU side for 10 minutes each followed by rinsing in neutral $\mathrm{pH}$ after each exposure. The scaffolds were continuously rinsed in phosphatebuffered solution ( $\mathrm{pH}$ 7.4) until the eluent $\mathrm{pH}$ was 7.4. The polymers were then functionalized with IKVAV and YIGSR to promote cellular functions important for the regeneration of bladder tissue. For this, standard aminophase silane chemistry was followed with $\mathrm{N}, \mathrm{N}-\left(3-\right.$ dimethylaminopropyl)- $\mathrm{N}^{\prime}$-ethyl-

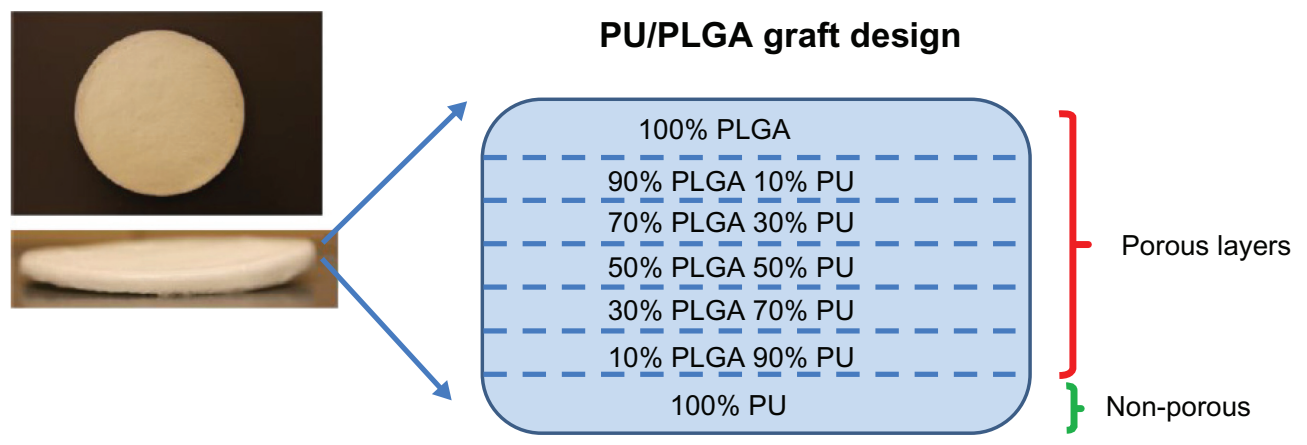

Figure I Design of the continuous novel graded PU-PLGA porous nanostructured graft. In the animal study, the $100 \%$ PLGA layer faced the urine while the I00\% PU layer faced the bladder lumen tissue.

Abbreviations: PU, polyurethane; PLGA, poly-lactic-co-glycolic acid. 
carbodiimide hydrochloride/N-hydroxysuccinimide (EDC/ NHS, Sigma, St Louis, MO, USA) used to immobilize IKVAV and YIGSR (Sigma). ${ }^{17}$ Lastly, radiofrequency glow discharge argon plasma was utilized for polymer scaffold sterilization for four minutes at $100 \mathrm{~W} .^{9}$

\section{Material characterization}

The topography and porosity of the nanostructured polymeric scaffolds were evaluated using scanning electron microscopy (JSM-840, JEOL, New York, NY, USA) at an accelerating voltage of $5 \mathrm{kV}$ and with Digital Scan Generator Plus software. Atomic force microscopy (NanoScope IIIa, Digital Instruments Inc, Boston, MA, USA) was also used to characterize surface roughness. Total porosity measurements were quantified using a SA $3100^{\mathrm{TM}}$ series surface area and pore size analyzer (Beckman Coulter, Fullerton, CA, USA) and the Brunauer-Emmett-Teller calculation. The chemistry of the polymers was characterized using x-ray photoelectron spectroscopy according to standard techniques.

The degradation rate of the nanostructured polymeric scaffolds was determined by placing them in cell buffer solutions (Dulbecco's Modified Eagle Medium, Gibco, St Louis, MO, USA) under standard incubator conditions for up to 23 days and calculating the weight retained after certain time periods. Lastly, the surface energy of each side of the samples was determined using sessile (static) water contact angles by placing $100 \mu \mathrm{L}$ of water onto each sample and using a camera to determine the contact angles.

\section{Mechanical properties}

\section{Burst pressure (ASTM D3787)}

To determine the ability of the bladder constructs to resist potential pressure increases representative of the bladder, a ball burst test was used. The pathological bladder wall tension range was found to be $9.8-11.7 \mathrm{~N} / \mathrm{cm}^{2}$ and the normal physiological bladder wall tension was found to be $2.8 \mathrm{~N} / \mathrm{cm}^{2} .{ }^{1}$ The ball burst strength is defined as the force that is required to burst the material when applied perpendicular to the plane of the material. A mechanical testing system 810 (MTS Co, Eden Prairie, MN, USA) was used with a burst strength fixture for knitted goods (ASTM 3787) to measure the biaxial strength of the bladder constructs. The burst strength fixture and the mechanical testing machine were used according to standard test methods for determining the bursting strength of textiles during a constant-rate-oftraverse ball burst. A polished steel ball was advanced at a constant rate (25.4 $\mathrm{mm}$ per minute) through the mesh. Eleven samples of each material were cut into $10 \mathrm{~cm} \times 5 \mathrm{~cm}$ each for testing. A mechanical burst pressure defined as between $9.8 \mathrm{~N} / \mathrm{cm}^{2}$ and $11.7 \mathrm{~N} / \mathrm{cm}^{2}$ will support the present design criteria for achieving favorable polymer graft characteristics for bladder applications. ${ }^{1}$

\section{Tensile test (ASTM D4I2)}

In order to measure the tensile strength of the polymer constructs, uniaxial tensile tests were also performed. The ultimate tensile strain for the human bladder has been reported as $0.69(\mathrm{~mm} / \mathrm{mm})$, the tensile strength as $0.27(\mathrm{MPa})$, and the elastic modulus as $0.25(\mathrm{MPa}) .{ }^{1}$ The samples were cut into dog-bone shapes $10 \mathrm{~cm}$ long and $2 \mathrm{~cm}$ wide in the middle. Constructs were clamped using a set of pneumatic piston clamps (Instron, Norwood, MA, USA) attached to the 810 mechanical testing system. The specimens were pulled at a rate of $25 \mathrm{~mm}$ per minute until failure. The force and displacement data were collected with computer software (Labview, Austin, TX, USA). Strength and stiffness were calculated from the stress-strain curves. Material thickness was assessed with a calibrated digital caliper (Mitutoyo, Tokyo, Japan). Eleven samples of each material were tested. A tensile strength defined as $0.52(\mathrm{MPa})$ or two times that of normal human bladder tensile strength will support the design criteria of achieving favorable polymer graft characteristics. ${ }^{1}$

\section{Suture pullout strength}

Lastly, polymer constructs were tested for suture retention by inserting four sutures into one end of each test sample, $2 \mathrm{~mm}$ from the end. Ethicon 2-0 Prolene ${ }^{\mathrm{TM}}$ sutures (Johnson and Johnson, Raynam, MA, USA) were used with an $\mathrm{SH}$ taper needle. The top pneumatic piston grip of the mechanical testing system was tightened to distribute the holding pressure equally along the surface of the sample. The sutures were inserted into the clamps of the lower grip, parallel to the direction of the force application. A mark made across the sample at the front inner edge of each grip was allowed, checking for sample slippage. Samples were tested at a constant rate of $25 \mathrm{~mm}$ per minute until the sample broke or until the suture material tore through the sample. Eleven samples of each material were tested. An adequate suture pullout strength defined as $25 \mathrm{~N}$ or better will support the present design criteria of achieving favorable graft characteristics. ${ }^{1}$

\section{In vivo study}

This part of the study tested the aforementioned PU-PLGA scaffolds for bladder augmentation in a partial cystoplasty model. All protocols were approved by the Brown University 
Institutional Animal Care and Use Committee per National Institutes of Health regulations. A total of eight female minipigs, divided into two groups (those receiving control ileal segments and those receiving the novel nanostructured synthetic grafts) at two time periods (five and 11 weeks) were used. Preoperatively, the animals were acclimatized for a minimum of two days before surgery. Oral antibiotics were administered preoperatively and continued for 10 days postoperatively. Each animal was rendered unconscious using an intramuscular injection of ketamine $15-20 \mathrm{mg} / \mathrm{kg}$ and xylazine $2 \mathrm{mg} / \mathrm{kg}$ and were then intubated and anesthetized with $1 \%-3 \%$ isoflurane. For the ileal segment reconstruction, a $12-18 \mathrm{~cm}$ segment of ileum located proximal to the ileocecal valve was measured and tagged by nondegradable sutures. The mesentery of the iliac segment selected was incised and prepared in a sequential manner using Kelly clamps and 3-0 free ties. The bowel segment was divided and the end of the ileal segment to be exteriorized was marked. Proximal and distal ends of the ileum were then anastomosed. The distal closed end of the ileal segment was excised and opened to allow copious irrigation of its lumen with saline solution. The ileal loop was oriented to allow peristalsis to proceed in the antegrade direction towards the cutaneous stoma. Next, the left and right ureters were isolated and anastomosed to the ileal segment. A circular skin excision in the previously marked stoma location was performed and an adequate crossed window was provided through the rectus fascia. The ileal end with a $2 \mathrm{~cm}$ nipple was secured to the rectus fascia with 3-0 Vicryl ${ }^{\mathrm{TM}}$ sutures (Ethicon). The stoma was then completed by folding the distal margin of the conduit to obtain a $1 \mathrm{~cm}$ nipple by suturing the mucosa to the skin with multiple interrupted 4-0 Vicryl sutures. Urethral catheters were left after surgery for seven days.

For the synthetic polymer scaffolds, the same process was followed, except for the harvesting of the ileal segment where the novel nanostructured polymer grafts were sutured rather than the ileal segment sutured into the bladder wall.

At time 0 and at the completion of the recovery period, urine and blood samples were collected and analyzed. After five and 11 weeks, the designated animals were euthanized and necropsy performed for harvesting the scaffolds and associated tissues for histological preparation and pathological examination. Standard hematoxylin and eosin stains were performed to assess bladder tissue generation and photographs were taken during biomaterial removal. Lastly, immediately after removal of the reconstructed bladder, the amount of water held by the reconstructed bladder was determined by filling the bladder with water until leakage by visual inspection. The amount of water held was then measured and reported as bladder capacity.

\section{Statistical analysis}

Quantitative data were analyzed using analysis of variance techniques with factorial designs. $P$ values $<0.05$ were considered to be statistically significant. All studies, unless otherwise noted, were run in triplicate per substrate type.

\section{Results and discussion \\ Material characterization}

When using a 9:1 salt to polymer ratio, the theoretical porosity of the PU-PLGA graft synthesized in the present study was $82.6 \%$. The measured porosity from scanning electron microscopic images was $87.6 \%$, which may be a result of the slightly larger amount of salt particles added during experiments. The size of the pores was determined by the size of the sieved salt particles which was between $150 \mu \mathrm{m}$ and $250 \mu \mathrm{m}$, as shown in Figure 2. Regardless, the porosity and pore size achieved meet the design criteria of creating a significant number of pores to increase bladder tissue growth and alter the mechanical properties to match that of bladder tissue. ${ }^{1}$

As mentioned, both the PU and PLGA sides of the graft were modified by chemical etching to possess nanometer rough surface features. The nontreated PU surface was very smooth without any noticeable features under $10 \mu \mathrm{m}$ (Figure 3A). In comparison, $\mathrm{HNO}_{3}$-etched $\mathrm{PU}$ had many submicron to nanometer rough pores on the

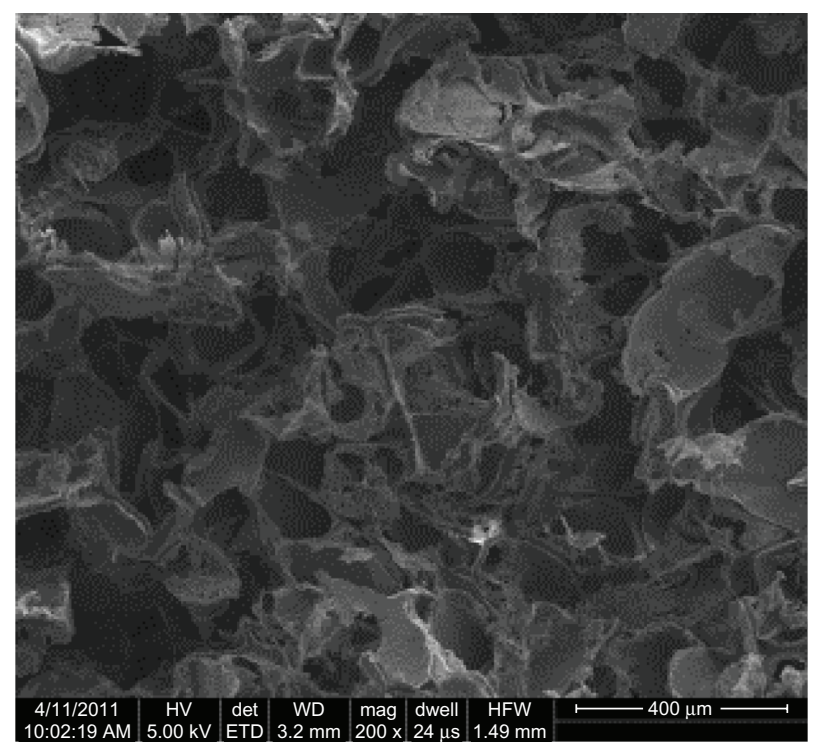

Figure 2 Porous structures of the PU-PLGA graft. Pore size was determined by sieved salt particle sizes, which had a size range of I50-250 $\mu \mathrm{m}$. Abbreviations: PU, polyurethane; PLGA, poly-lactic-co-glycolic acid. 


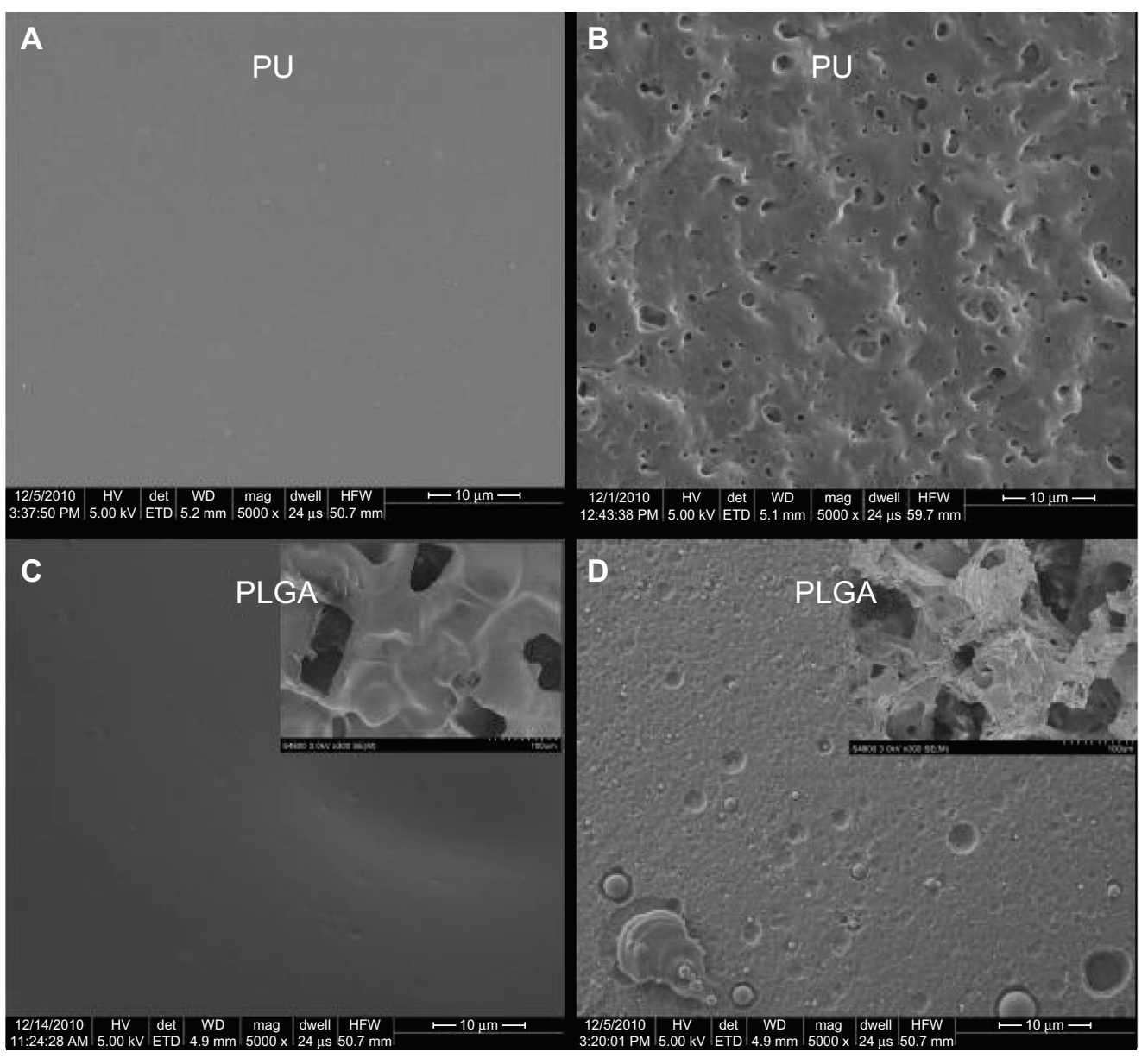

Figure 3 Scanning electron microscopic images of $(\mathbf{A})$ PU control, $(\mathbf{B})$ nanomodified PU, (C) PLGA control, and (D) nanomodified PLGA. Scale bars = I0 $\mu$ m. Insets in (C) and (D) are unmodified and nanomodified porous PLGA layers under low magnification, respectively.

Abbreviations: PU, polyurethane; PLGA, poly-lactic-co-glycolic acid.

surface (Figure 3B). Similarly, the PLGA surfaces were modified by soaking in $\mathrm{NaOH}$ to have nanometer rough surface features (Figure 3C and D). From the images, as expected, it is obvious that some PLGA was etched away by the chemical treatment. Thus, it was clear that the present PU-PLGA composites possessed the desired nanoscale surface features previously shown to promote bladder cell responses. ${ }^{1}$ Atomic force microscopic images (Figure 4) and roughness analysis (Figure 5) further confirmed the qualitative findings from scanning electron microscopy that the nanotreated PLGA and PU were much more rough at the nanoscale than their original surfaces, respectively.

As a result of this change in surface roughness at the nanoscale, as expected, the chemically etched PU showed greater hydrophobicity (transitioning from contact angles of 74-85 degrees, Figure 6). In contrast, the flat PLGA was hydrophobic (around 101 degrees) while the porous (150$250 \mu \mathrm{m}$ ) nanorough PLGA was hydrophilic (84 degrees). Such results are expected due to the ether bond breakage in
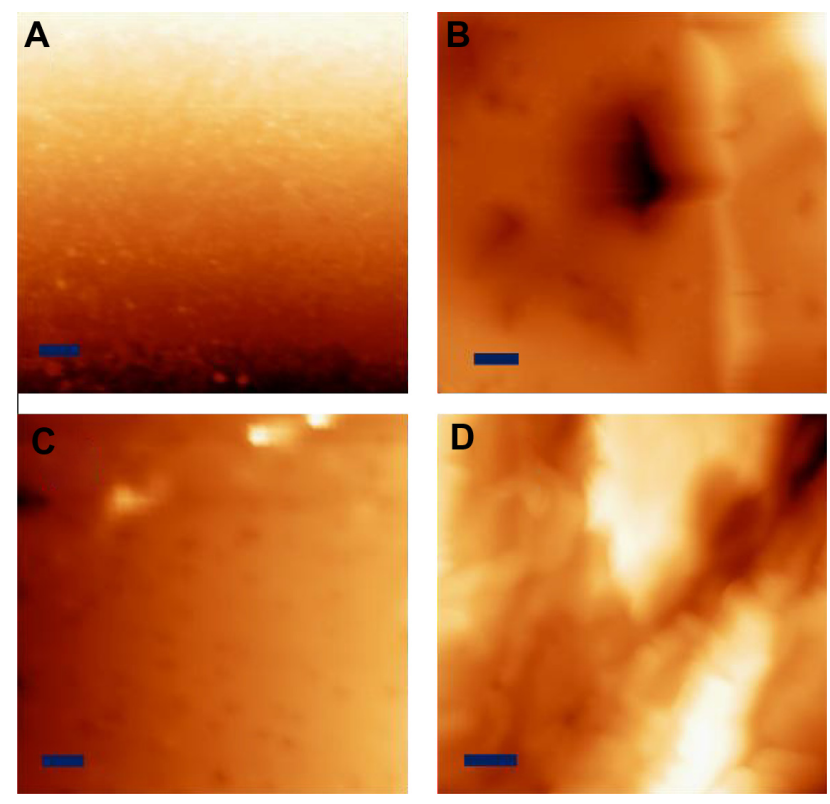

Figure 4 Atomic force micrographs of (A) conventional PU, (B) nanomodified PU, (C) conventional PLGA, and (D) nanomodified PLGA. Scale bars $=I \mu \mathrm{m}$. Abbreviations: PU, polyurethane; PLGA, poly-lactic-co-glycolic acid. 


\section{AFM RMS roughness}
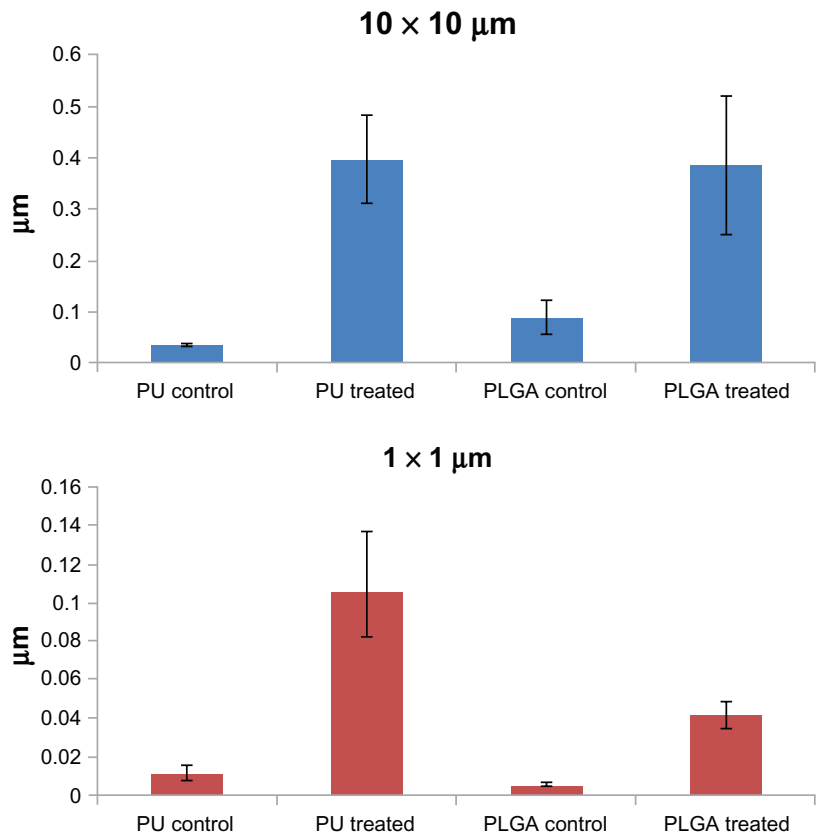

Figure 5 RMS roughness of PU and PLGA polymers at different scales. Abbreviations: AFM, atomic force microscopy; PU, polyurethane; PLGA, polylactic-co-glycolic acid; RMS, root mean square.

PU and ester bond breakage in PLGA. The reference material, ie, small intestinal submucosa, had a water contact angle about 60 degrees. ${ }^{16}$

Lastly, x-ray photoelectron spectroscopy was used to confirm covalent bonding of the two peptides of interest in the present study, IKVAV and YIGSR, onto the polymer surfaces (Table 1). With the presence of only EDC and NHS, there was no significant increase in nitrogen content after an hour of soaking with polymer graft and rinsing afterwards (Table 1, $0.2 \%$ for PLGA and $0.3 \%$ for PLGA soaked with EDC/NHS). In comparison, with the presence of both EDC/NHS and the abovementioned peptides, there was a significant increase in nitrogen content $(1.3 \%)$ on the polymer surface after an

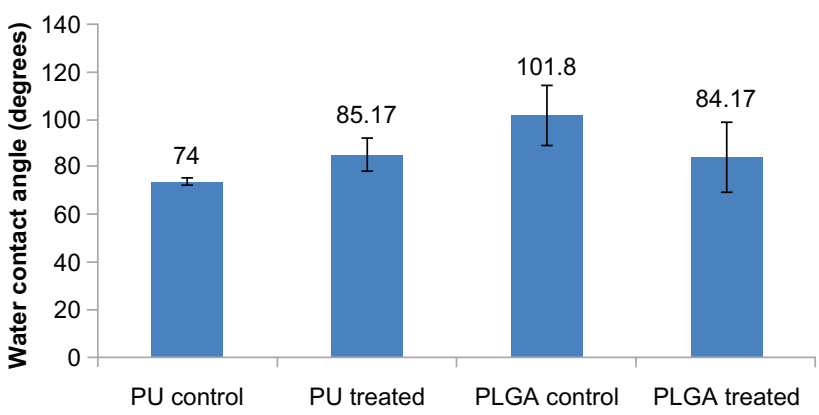

Figure 6 Water contact angles for PU and PLGA surfaces before and after surface modification.

Abbreviations: PU, polyurethane; PLGA, poly-lactic-co-glycolic acid.
Table I X-ray photoelectron spectroscopic analysis of compositions on peptide-functionalized PLGA showing the same results for peptides

\begin{tabular}{llllll}
\hline Sample & C Is\% & N Is\% & O Is\% & S 2p\% & Si 2p\% \\
\hline PLGA & 64.6 & 0.2 & 34.9 & 0.1 & 0.2 \\
PLGA + EDC/NHS & 64.0 & 0.3 & 35.6 & 0.0 & 0.1 \\
PLGA + EDC/NHS + PEPTIDE & 64.7 & 1.3 & 33.4 & 0.1 & 0.5 \\
\hline
\end{tabular}

Abbreviations: $\mathrm{EDC}, \mathrm{N}, \mathrm{N}$-(3-dimethylaminopropyl)- $\mathrm{N}^{\prime}$-ethyl-carbodiimide hydrochloride; NHS, N-hydroxysuccinimide; PLGA, poly-lactic-co-glycolic acid.

hour of reaction and rinsing afterwards, indicating that both peptides were present.

\section{Graft mechanical strength}

Equally as important to determining material surface properties of the proposed PU-PLGA scaffolds, it is critical to determine their mechanical properties for assessment of their suitability for application in bladder tissue regeneration. For this, as mentioned, the mechanical strength of the graft materials was assessed using three mechanical tests (tensile, ball burst, and suture pullout) and carried out using the aforementioned American Society of Testing Material standards. The results showed that the tensile strength of the seven-layer PU-PLGA construct was about $0.71 \mathrm{MPa}$, which was two times higher than normal bladder tissue strength $(0.54 \mathrm{MPa})$. When compared with multilayered small intestinal submucosa, the synthetic polymer scaffold showed similar load at yield but a lower stiffness (slope of curve). ${ }^{16}$ It is noted that there were small drops along the curve that could be indications of PU-PLGA layer breakage (Figure 7). Moreover, the PU-PLGA polymer graft showed maximum force similar to that of four-layer small intestinal submucosa material in the ball burst and suture pullout tests (Figures 8 and 9). ${ }^{16}$ Again, the polymer graft showed much larger displacement and, thus, lower stiffness. In summary, the mechanical tests provided evidence of mechanical properties (tensile, ball burst, and suture pullout) similar to those of natural bladder tissue and small intestinal submucosa (Table 2) ${ }^{16}$

\section{Degradation}

The results of the present study also provide evidence of similar degradation properties between nanomodified PLGA-PU and control composites for up to 23 weeks, with approximately $40 \mathrm{wt} \%$ degradation for both materials after 23 weeks (Figure 10).

\section{Blood and urine tests}

As mentioned, urine and blood was collected from animals with either the ileal segment or polymer graft the day 


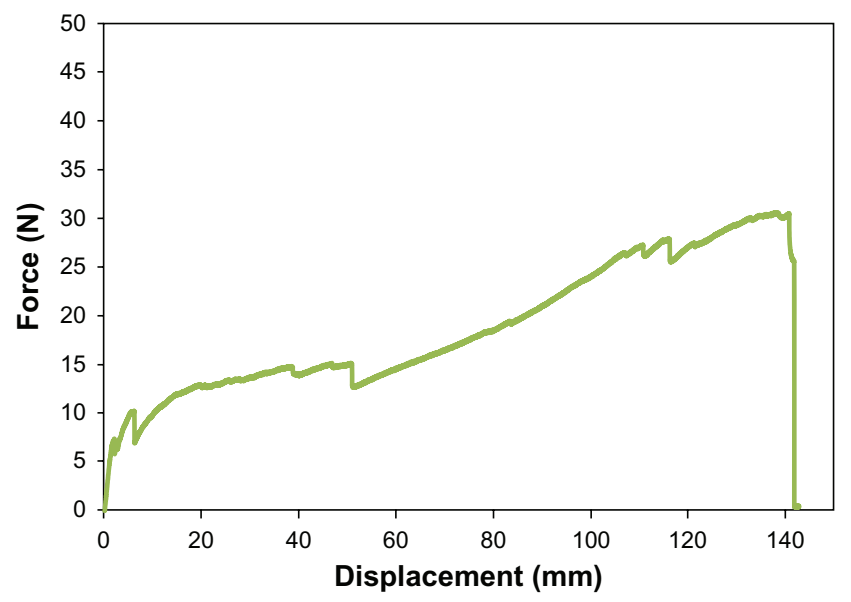

Figure 7 Tensile test plot of PU-PLGA grafts (left) compared with single and multilayered SIS grafts. ${ }^{16}$

Abbreviations: PU, polyurethane; PLGA, poly-lactic-co-glycolic acid; SIS, single layered small intestinal submucosa; MLSIS, multilayered small intestinal submucosa.

after surgery and before sacrifice five and 11 weeks later (Tables 3 and 4). For urinalysis, the four animals (labeled as $03-38$ and $03-24$ for the five-week implantation time and 00-18 and 00-89 for the 11-week implantation time) with the ileal grafts all had yellow hazy urine at a $\mathrm{pH}$ of around 6.5 the day after surgery. The 03-24 animal showed a trace of blood and a few red blood cells. The 00-18 animal showed a few white blood cells, a few bacteria, and a few epithelial cells. The 00-89 animal showed a few white blood cells and a few epithelial cells. After five weeks, the urinary $\mathrm{pH}$ was 7.5 or 8 . The $03-38$ animal showed blood, a moderate number of white blood cells, a moderate number of red blood cells, and a moderate number of epithelial cells. The other animal (03-24) showed a moderate number of white blood cells, a moderate number of epithelial cells, and a moderate

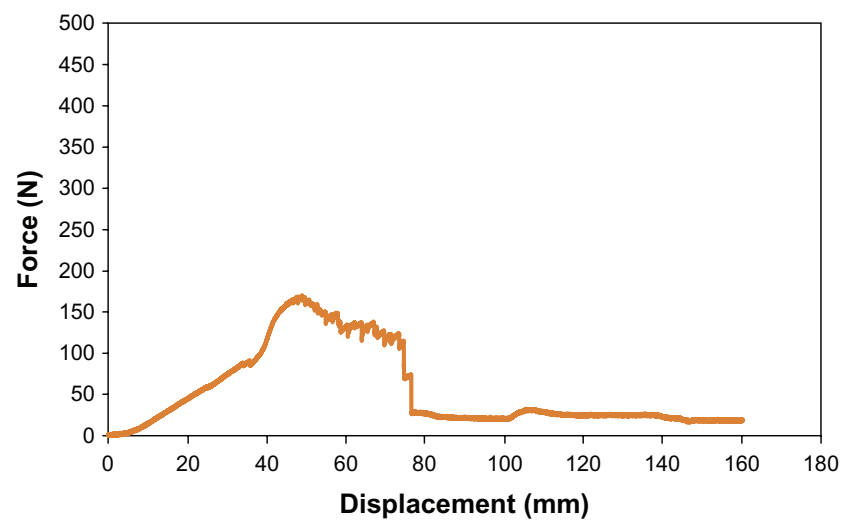

Figure 8 Ball burst test plot of PU-PLGA grafts (left) compared with single and multilayered SIS grafts. ${ }^{16}$

Abbreviations: PU, polyurethane; PLGA, poly-lactic-co-glycolic acid; SIS, single layered small intestinal submucosa; MLSIS, multilayered small intestinal submucosa. amount of oxalate crystals. After 11 weeks, the $00-18$ animal showed a trace of blood, a few white blood cells, a moderate number of red blood cells, and a few epithelial cells. The 00-89 animal showed a trace of blood, a marked number of white blood cells, a moderate number of red blood cells, and a moderate number of epithelial cells.

The four animals (labeled $00-40$ and $06-13$ for the fiveweek implantation time and $03-41$ and $03-27$ for the 11-week implantation time) with polymer grafts also had yellow hazy urine at a $\mathrm{pH}$ of 6.5-7.5 the day after surgery. The 03-41 animal showed a few white blood cells and a few epithelial cells. The 03-27 animal showed a trace amount of blood, a few white blood cells, a moderate number of red blood cells, a marked number of bacteria, a few epithelial cells, and a few crystals in the urine samples. After five weeks, the 06-13 animal showed blood, a marked number of white blood cells, a moderate number of red blood cells, a moderate number of bacteria, a moderate number of epithelial cells, and a marked number of crystals in the urine. After 11 weeks, the 03-41 animal showed a trace of blood, a moderate number of white blood cells, a few red blood cells, and a few epithelial cells. The 03-27 animal showed a moderate number of white blood cells, a moderate number of bacteria, a few epithelial cells, and moderate crystal formation. In summary, the results of urine tests showed no major differences between the use of an ileal segment versus a polymer graft.

Comparing the presence of immune cells in the blood between the animals receiving the ileal segment or the PLGAPU nanocomposite, animals with polymer grafts had neutrophil, lymphocyte, and monocyte levels similar to or lower than those in animals with an ileal graft at the three different time

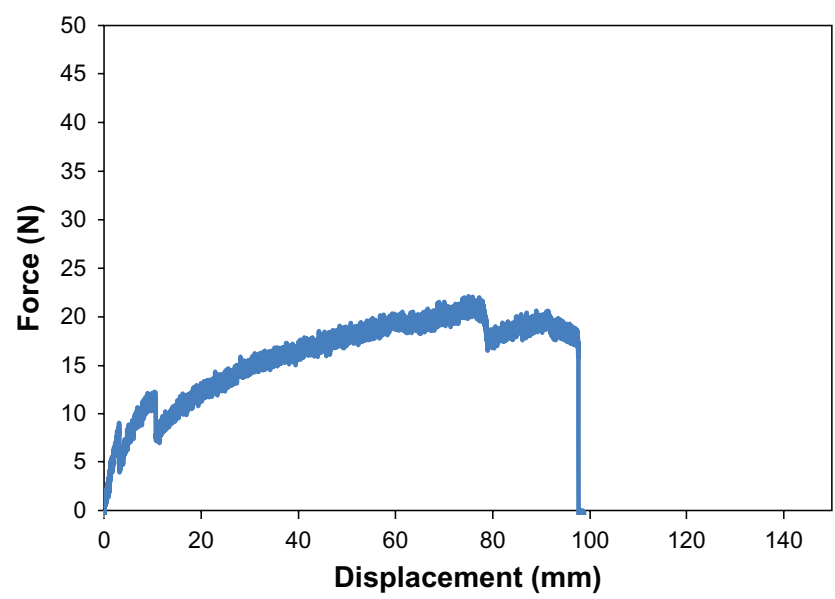

Figure 9 Suture pullout test plot of PU-PLGA grafts (left) compared with single and multilayered SIS grafts. ${ }^{16}$

Abbreviations: PU, polyurethane; PLGA, poly-lactic-co-glycolic acid; SIS, single layered small intestinal submucosa; MLSIS, multilayered small intestinal submucosa. 
Table 2 Summary of mechanical testing of proposed nanostructured PU-PLGA graft versus design criteria

\begin{tabular}{lll}
\hline & Design criteria & Result \\
\hline Suture & I4-100 N (I4 N for a 4 layer porcine & $21 \pm 5 \mathrm{~N}$ \\
pull out & small intestine submucosa) & \\
Tensile & $\begin{array}{l}2 \times \text { normal human bladder strength } \\
\text { or } 0.54 \mathrm{MPa}\end{array}$ & $0.71 \pm 0.15 \mathrm{MPa}$ \\
Ball burst & $>9.8-11.7 \mathrm{~N} / \mathrm{cm}^{2}$ (pathological BWT) & $22.1 \mathrm{~N} / \mathrm{cm}^{2}$ \\
\hline
\end{tabular}

Abbreviations: BWT, bladder wall tension; Mpa, mega pascals; N, Newtons; PU, polyurethane; PLGA, poly-lactic-co-glycolic acid.

points ( 0 , five, and 11 weeks). For both groups, blood collected the day after surgery showed a higher number of neutrophils than that collected after five or 11 weeks. The number of reticulocytes and platelets showed no obvious trends. In summary, blood tests provided evidence of a stronger immune reaction (due to the presence of more immune cells in the blood) when using the ileal segment compared with the polymer grafts; of note, use of an ileal segment as a bladder replacement material required two separate surgeries which clearly increases the chance of an immune response.

There was one animal in the ileal segment group that showed signs of contamination in the urine the day after surgery. In addition, one animal in the nanostructured polymer graft group showed signs of contamination the day after surgery and moderate contamination after 11 weeks. Another animal with the polymer graft showed moderate contamination after five weeks. The appearance of bacteria may be attributed to either contamination during the procedures or, less probably, endotoxins inside the graft. The plasma sterilization method used in this study is recognized by different researchers but has not been validated for the present specific bladder regeneration application. It is important to note that previous in vitro studies have demonstrated less bacteria adhesion and growth on nanostructured PLGA and

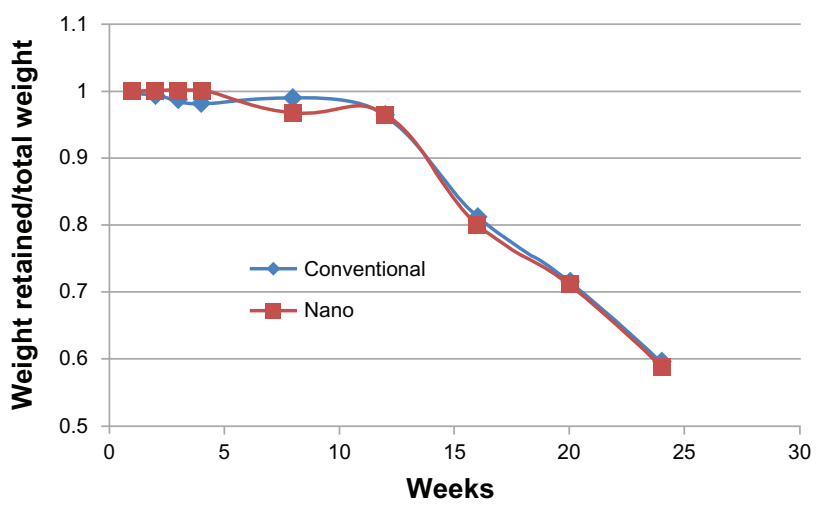

Figure 10 Degradation of conventional and nanomodified PU-PLGA constructs in buffer solution over 24 weeks.

Abbreviations: PU, polyurethane; PLGA, poly-lactic-co-glycolic acid.
PU compared with conventional PLGA and PU as prepared in this study.

\section{Bladder capacity}

The ileal segment and polymer grafts were able to withstand the same pressure after five and 11 weeks of implantation (40 $\mathrm{cm} \mathrm{H}_{2} \mathrm{O}$, Table 5). The average bladder capacity of the minipigs which underwent ileal segment augmentation was $425 \mathrm{~mL}$ after five weeks and $630 \mathrm{~mL}$ after 11 weeks. Importantly, the average bladder capacity of minipigs who underwent polymer graft augmentation was $295 \mathrm{~mL}$ after five weeks and $645 \mathrm{~mL}$ after 11 weeks, showing equivalence with the ileal segment group after 11 weeks.

\section{Integration of ileal segments and polymer grafts into the bladder}

Most importantly, macroscopic images (Figure 11) clearly showed that the ileal segments remained attached and viable in the resected portion of bladder wall after five and 11 weeks. Polymer grafts appeared to have partially formed tissue after five weeks and fully formed tissue across the entire resected area after 11 months. The estimated regenerated bladder area initially replaced by the ileal tissue segment and the polymer graft was $4 \mathrm{~cm} \times 4 \mathrm{~cm}$. Consistent with the observed equivalence of bladder capacity between the two groups after 11 weeks, the transplanted mucosal tissue of the ileal segment was estimated at $4 \mathrm{~cm} \times 4 \mathrm{~cm}$ at 11 weeks while a $4 \mathrm{~cm} \times 4 \mathrm{~cm}$ area of new bladder tissue had formed on the polymer graft after 11 weeks. Interestingly, after 11 weeks, a thin residual PU layer had started to separate from the newly formed bladder tissue. The thin residual PU layer was easily separated from the newly formed bladder tissue by gently pulling the PU.

The thickness of the PU-PLGA polymer grafts decreased from around $2.5 \mathrm{~mm}$ to $0.5 / 0.3 \mathrm{~mm}$ after five weeks and to $0.3 / 0.3 \mathrm{~mm}$ after 11 weeks. Because the estimated thickness of the $100 \%$ PU layer was about $0.2 \mathrm{~mm}$, it is reasonable to conclude that most of the PLGA components in the graft had been degraded after 11 weeks. Clearly, hydrolysis of PLGA in vivo is much faster than that in vitro. The nanostructured polymer graft lost most of its thickness after 11 weeks in the minipig but maintained the majority of its mass in vitro. This is due to the significant difference in conditions in the bladder (ie, dynamic fluid flow and composition of fluid) compared with the static in vitro testing conditions used. However, when compared with small intestinal submucosa, the PU-PLGA behaved similarly; that is, less than $10 \%$ of small intestinal submucosa remained three months after implantation in a pig 
Table 3 Urinalysis of ileal-grafted and polymer-grafted animals

\begin{tabular}{|c|c|c|c|c|c|c|c|c|c|}
\hline Graft & Label & Time & Blood & WBC & RBC & EPI cell & Bacteria & Crystal & $\mathrm{pH}$ \\
\hline \multirow[t]{4}{*}{ Ileal } & $03-38$ & $0 \mathrm{~W}$ & 0 & 0 & 0 & 0 & 0 & 0 & 6.5 \\
\hline & & $5 W$ & $3+$ & $2-5$ & $2-5$ & $1-2$ & 0 & 0 & 8 \\
\hline & $03-24$ & $0 \mathrm{~W}$ & Trace & 0 & $0-2$ & 0 & 0 & 0 & 6.5 \\
\hline & & $5 W$ & 0 & $2-5$ & 0 & $3-5$ & 0 & $3-5$ & 7.5 \\
\hline \multirow[t]{4}{*}{ Polymer } & $00-40$ & $0 \mathrm{~W}$ & 0 & 0 & 0 & 0 & 0 & 0 & 6 \\
\hline & & $5 \mathrm{~W}$ & I & I & I & I & I & I & 1 \\
\hline & $06-13$ & $0 \mathrm{~W}$ & 0 & 0 & 0 & 0 & 0 & 0 & 7 \\
\hline & & $5 W$ & I+ & $10-15$ & $2-5$ & $\mathrm{I}-2$ & $9-40$ & $6-10$ & 7.5 \\
\hline \multirow[t]{4}{*}{ Ileal } & $00-18$ & $0 \mathrm{~W}$ & 0 & $0-2$ & 0 & $0-1$ & $<8$ & 0 & 6 \\
\hline & & II W & Trace & $0-2$ & $2-5$ & $0-1$ & 0 & 0 & 6 \\
\hline & 00-89 & $0 \mathrm{~W}$ & 0 & $0-2$ & & $0-1$ & 0 & 0 & 6.5 \\
\hline & & II W & Trace & $20-30$ & $2-5$ & $3-5$ & 0 & 0 & 6.5 \\
\hline \multirow[t]{4}{*}{ Polymer } & $03-41$ & $0 \mathrm{~W}$ & 0 & $0-2$ & 0 & $0-1$ & 0 & 0 & 6.5 \\
\hline & & II W & Trace & $2-5$ & $0-2$ & $0-1$ & 0 & 0 & 6 \\
\hline & $03-27$ & $0 \mathrm{~W}$ & Trace & $0-2$ & $2-5$ & $0-1$ & $>40$ & $0-1$ & 7.5 \\
\hline & & II W & 0 & $2-5$ & 0 & $0-1$ & $9-40$ & $3-5$ & 8 \\
\hline
\end{tabular}

Notes: Unit for urinalysis is per high power field, refers to the area visible under the maximum magnification power of the objective; “l”, not available due to sample loss. Abbreviations: RBC, red blood cells; WBC, white blood cells; EPI cell, epithelial cells; W, weeks.

study ${ }^{6}$ while $40 \%$ remained after one month with complete loss after three months in a canine study. ${ }^{7}$

Of intrigue, is the separation of the nanostructured PU-PLGA graft from the bladder wall during bladder wall regeneration. Of course, this may allow for easy removal of the graft after integration (for example, using laparoscopy); however, care will need to be taken to match regeneration time carefully with graft removal. Clearly, future studies will be needed to take full advantage of this novel property of nanostructured PU-PLGA grafts to separate from bladder tissue after bladder tissue regeneration.

\section{Histological analysis of urothelial and smooth muscle tissue regeneration}

On histological examination, the transplanted ileal segment itself appeared to integrate well into the bladder tissue after five and 11 weeks of implantation (Figures 12 and 13). Specifically, after five weeks, a layer of urothelium was observed across the site of the nondegradable sutures used. However, no smooth muscle cell layer had formed after five weeks. After 11 weeks, both urothelium and smooth muscle formed on the newly created bladder tissue when using the ileal segment. The remodeling process between the original ileal epithelial cells and the regenerated urothelial cells is unclear and will require further experiments.

In contrast, after five weeks, both urothelial and smooth muscle cell layers formed on the nanostructured polymer graft (Figures 12 and 13). Remnants of the polymeric material (one micron or less) were observed next to the newly formed bladder walls, suggesting successful incorporation of new tissue into the pores of the graft. The composition of the polymer observed in the histological segments is not known, and will need to be determined in future studies. If it is residual PLGA (as expected), the polymer remnants would be expected to degrade completely with sufficient time. After 11 weeks, both the urothelium and smooth muscle were con-

Table 4 Selected cells detected in blood samples from animals with an ileal or polymer graft

\begin{tabular}{|c|c|c|c|c|c|}
\hline & Neutrophil & Lymphocyte & Monocyte & Reticulocyte & Platelet \\
\hline \multicolumn{6}{|c|}{ Ileal segment } \\
\hline $0 \mathrm{~W}$ & 7213 & 5686 & 455 & 40018 & 599000 \\
\hline $5 W$ & 3680 & 5622 & 549 & 19330 & 422000 \\
\hline $11 \mathrm{~W}$ & 5314 & 6419 & 618 & 14000 & 715500 \\
\hline \multicolumn{6}{|c|}{ Nano polymer } \\
\hline $0 \mathrm{~W}$ & 6522 & 4856 & 248 & 15263 & 473000 \\
\hline $5 \mathrm{~W}$ & 4177 & 4985 & 397 & 38595 & 442000 \\
\hline II W & 2784 & 5481 & 348 & I & 338000 \\
\hline
\end{tabular}

Notes: $0 W(n=4), 5 W(n=2)$, and II W $(n=2) . /=$ negligible.

Abbreviation: $\mathrm{W}$, weeks. 
Table 5 Capacity of bladder with an ileal segment graft or polymer graft after five and II weeks $(n=2)$

\begin{tabular}{lll}
\hline & $\mathbf{5}$ weeks & I I weeks \\
\hline Augmentation by ileal segment & $425 \mathrm{cc}$ & $630 \mathrm{cc}$ \\
Augmentation by nano polymer graft & $295 \mathrm{cc}$ & $645 \mathrm{cc}$ \\
\hline
\end{tabular}

Note: Original average bladder capacity in all pigs was $630 \mathrm{cc}$. Abbreviation: cc, milliliters.
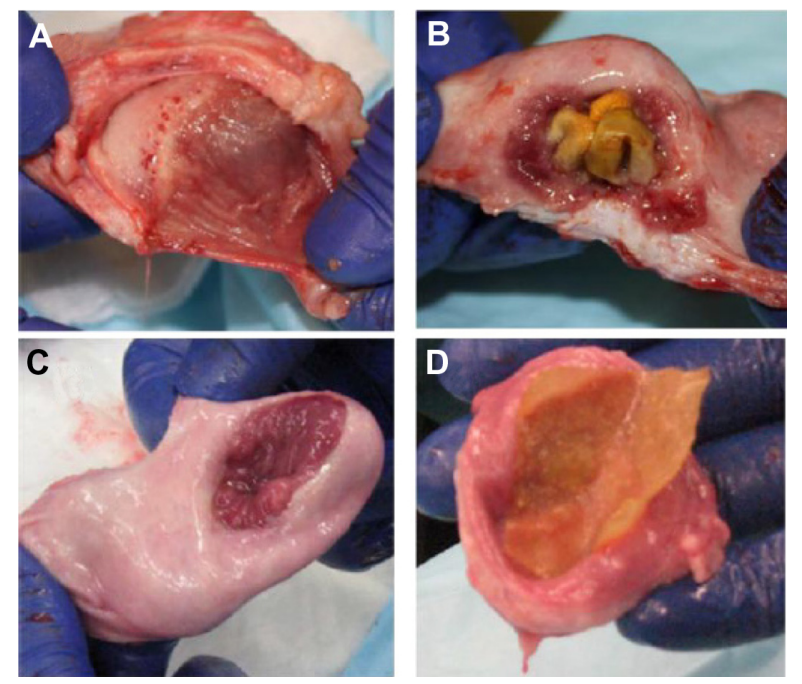

Figure II Observation of ileal and polymer grafts five and II weeks after implantation. (A) lleal graft at five weeks, (B) polymer graft at five weeks, (C) ileal graft at II weeks, and (D) polymer graft at II weeks. The polyurethane layer was easily separated from the newly formed bladder tissue with a gentle pull. sistently and continuously formed on the new bladder wall tissue with the incorporated polymer present.

\section{Conclusion}

A synthetic polymer graft comprised of nanostructured PU and nanostructured PLGA was designed for partial bladder replacement. The graft was fabricated to have porosity to support infiltration of bladder tissue into all sides of the graft. Both polymer surfaces were modified to possess nanostructured features and with IKVAV and YIGSR to enhance their interactions with cells. Mechanical tests confirmed that this polymer graft has appropriate mechanical properties for bladder wall replacement. This synthetic PU-PLGA nanostructured graft was assessed in a well established minipig model, and showed similar bladder capacity, similar regeneration size, and similar tissue microstructure for newly formed bladder tissues up to 11 weeks between the ileal segment and polymer graft. The PLGA matrix portion of the graft was fully desorbed and a thin residual PU layer which could be gently peeled away from the new bladder wall was observed. Taken together, these findings support the continued development of this nanostructured resorbable synthetic graft for partial bladder and urogenital repair as well as neobladder replacement. Thus, this synthetic PU-PLGA
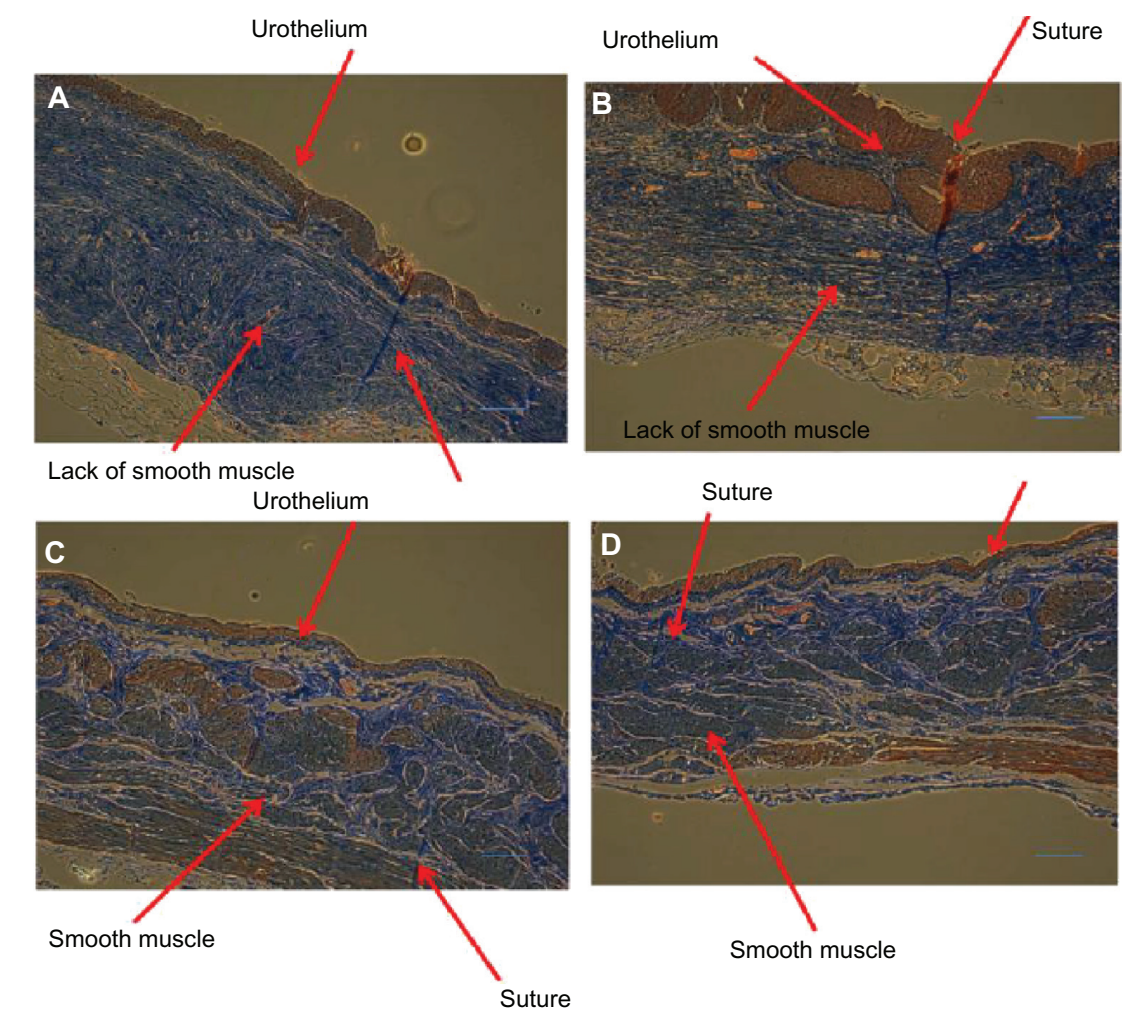

Figure 12 lleal segment histology showing newly formed urothelial layers after five weeks (A and $\mathbf{B})$ and formation of both urothelial and smooth muscle tissue after II weeks (C and $\mathbf{D})$ on the transplanted ileal segment. Scale bar $=1 \mu \mathrm{m}$. 

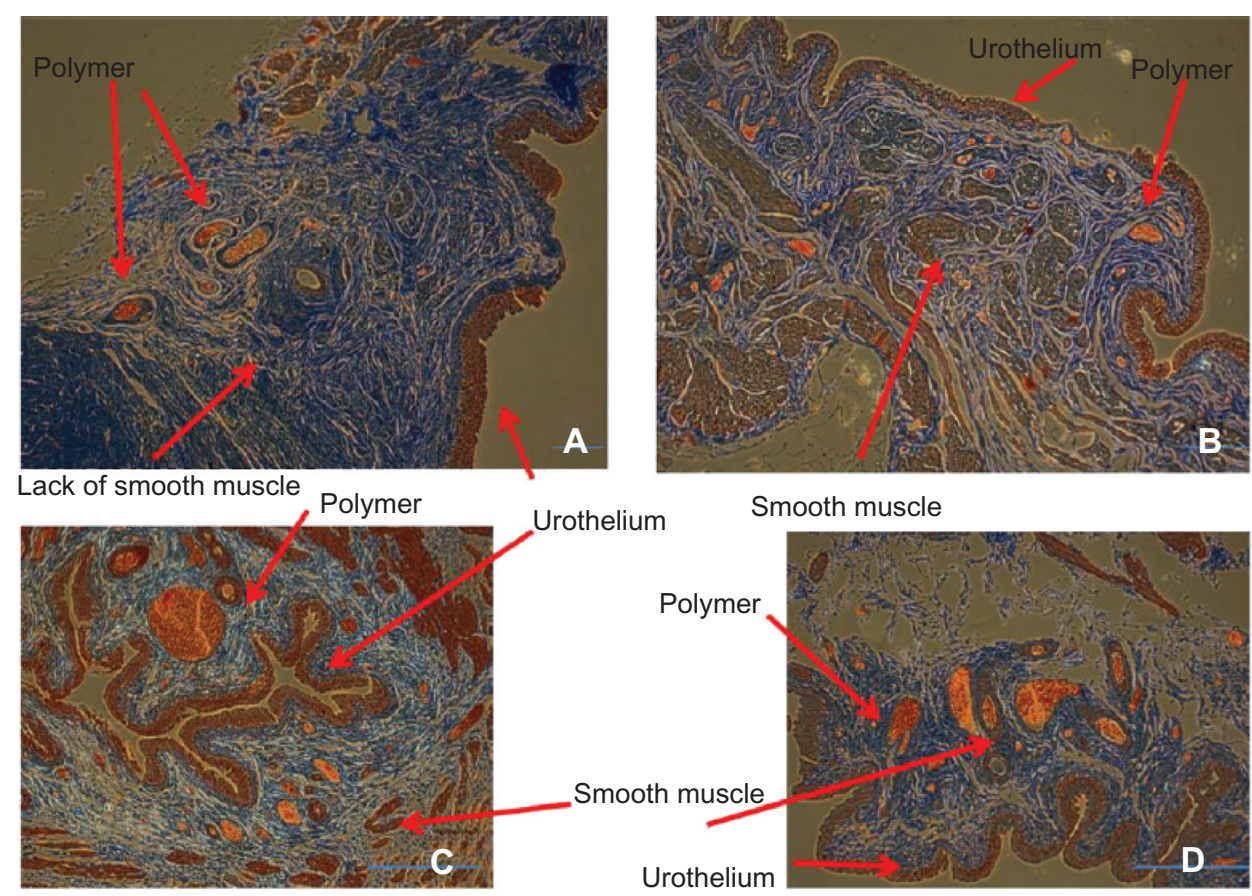

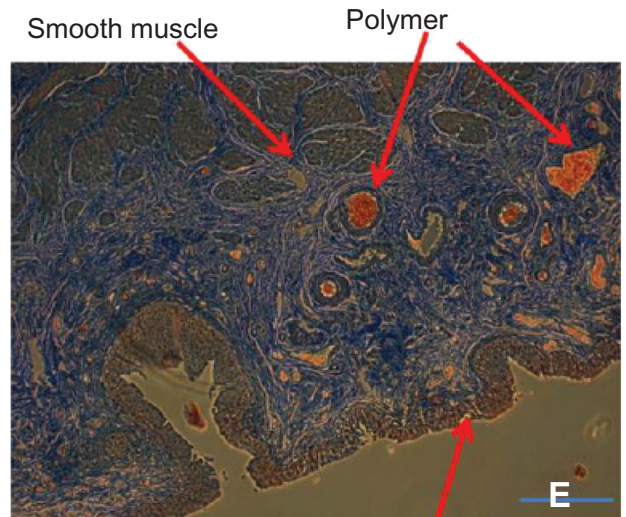

Urothelium

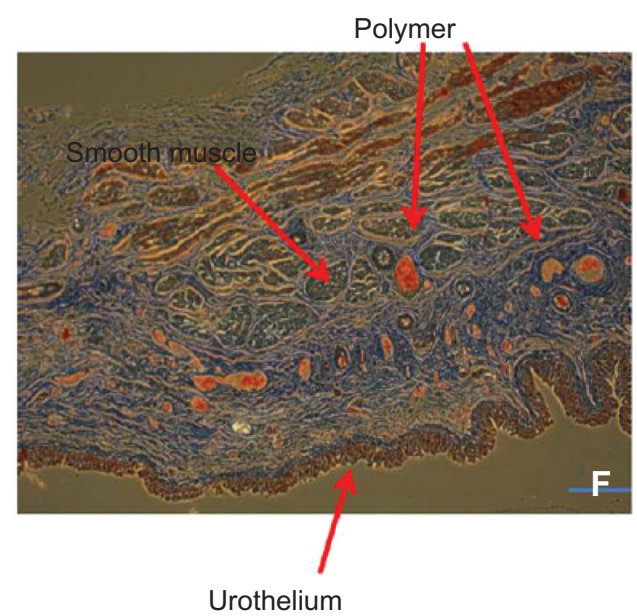

Figure 13 Nanomodified polymer graft histology showing newly formed urothelial and smooth muscle layers after five weeks (A-D) and continued maturation of urothelial and smooth muscle tissue after II weeks $(\mathbf{E}$ and $\mathbf{F})$ on the newly formed tissue around the nanomodified polymer graft. Scale bar $=$ I $\mu$ m.

nanostructured composite offers significant new clinical options for the treatment of patients suffering from numerous bladder problems, including congenital defects, bladder cancer, and other bladder trauma.

\section{Acknowledgment}

The authors would like to acknowledge the National Cancer Institute of the National Institutes of Health for financially supporting the present study (grant 1R41CA136033-01A2).

\section{Disclosure}

The authors CY, MH, and TJW are employees of Nanovis LLC, IN, USA. All other authors declare no conflicts of interest in this work.

\section{References}

1. Thapa A, Webster TJ, Haberstroh KM. Nano-structured polymers enhance bladder smooth muscle cell function. Biomaterials. 2003;24: 2915-2926.

2. Thapa A, Webster TJ, Haberstroh KM. Nano-structured polymers enhance bladder smooth muscle cell adhesion. J Biomed Mater Res. 2003;67A:1374-1383.

3. Tanzi MC, Mantovani D, Petrini P, Guidoin R, Laroche G. Chemical stability of polyether urethanes versus polycarbonate urethanes. J Biomed Mater Res. 1997;36:550-559.

4. Tanzi MC, Fare S, Petrini P. In vitro stability of polyether and polycarbonate urethanes. J Biomater Appl. 2000;14:325-348.

5. Fare S, Petrini P, Motta A, Cigada A, Tanzi MC. Synergistic effects of oxidative environments and mechanical stress on in vitro stability of polyetherurethanes and polycarbonateurethanes. J Biomed Mater Res. 1999;45:62-74.

6. Miller DC, Thapa A, Haberstroh KM, Webster TJ. Enhanced functions of vascular cells on nanostructured polymers. Biomaterials. 2004;25: $53-61$. 
7. Vance R, Miller D, Thapa A, Haberstroh K, Webster TJ. Decreased fibroblast cell density on chemically degraded poly-lactic-glycolic acid, polycaprolactone, and polyurethane. Biomaterials. 2004;25: 2095-2103.

8. Kay S, Thapa A, Haberstroh KM, Webster TJ. Nanostructured polymer: nanophase ceramic composites enhance osteoblast and chondrocyte adhesion. Tissue Eng. 2002;8:753-761.

9. Machado M, Tarquinio K, Webster TJ. Decreased S. aureus biofilm formation on nanomodified ETTs: an airway dynamic model. Int $J$ Nanomedicine. 2012;7:3741-3750.

10. Geilich B, Webster TJ. Reduced adhesion of Staphylococcus aureus to ZnO/PVC nanocomposites. Int J Nanomedicine. 2013;8:1-8.

11. Holton PD, Shinar Z, Benna J, Patzakis MJ. Porcine small intestine submucosa does not show antimicrobial properties. Clin Orthop Relat Res. 2004;427:18-24.

12. Chun YW, Khang D, Haberstroh KM, Webster TJ. The role of polymer nanosurface roughness and submicron pores in improving bladder urothelial cell density and inhibiting calcium oxalate stone formation. Nanotechnology. 2009;20:085104.
13. Taylor E, Webster TJ. Reducing infections through nanotechnology and nanoparticles. Int J Nanomedicine. 2011;6:1463-1473.

14. Jernigan TW, Croce MA, Cagiannos C, Shell DH, Handorf CR, Fabian TC. Small intestinal submucosa for vascular reconstruction in the presence of gastrointestinal contamination. Ann Surg. 2004;239: 733-738.

15. Yao C, Hedrick M, Webster T. Decreased bacteria adhesion and growth on nanostructured polymers. Int J Nanomedicine. In press.

16. Arnold GA, Mathews KG, Roe S, Mente P, Seaboch T. Biomechanical comparison of four soft tissue replacement materials: an in vitro evaluation of single and multilaminate porcine small intestinal submucosa, canine fascia lata, and polypropylene mesh. Vet Surg. 2009;38:834-844.

17. Danczyk RC, Krieder B, North A, Webster TJ, Hogenesch H, Rundell A. Comparison of antibody functionality using different immobilization methods. Biotechnol Bioeng. 2003;84:215-223.
International Journal of Nanomedicine

\section{Publish your work in this journal}

The International Journal of Nanomedicine is an international, peerreviewed journal focusing on the application of nanotechnology in diagnostics, therapeutics, and drug delivery systems throughout the biomedical field. This journal is indexed on PubMed Central, MedLine, CAS, SciSearch ${ }^{\circledR}$, Current Contents ${ }^{\circledR} /$ Clinical Medicine,

\section{Dovepress}

Journal Citation Reports/Science Edition, EMBase, Scopus and the Elsevier Bibliographic databases. The manuscript management system is completely online and includes a very quick and fair peer-review system, which is all easy to use. Visit http://www.dovepress.com/ testimonials.php to read real quotes from published authors. 